CASE REPORT

\title{
An Atypical Soft Tissue Mass on the Dorsum of the Foot: A Case Report
}

\author{
Kirmanj Atrushi PGY-3, DPM \\ Atlanta Veterans Affairs Medical Center, USA
}

*Corresponding author: Kirmanj T Atrushi, PGY-3, DPM, Atlanta Veterans Affairs Medical Center, 1670 Clairmont Road, Decatur, GA 30033, USA, Tel: (224)-440-1927

\begin{abstract}
Evaluating and treating soft tissue masses is a component of podiatric practice that few will be able to avoid. Indeed, of all soft tissue masses, ganglion cysts appear to be the most common. Many of these can be adequately treated with fine-needle aspiration. However, in other cases, surgical excision may be indicated, particularly when the cyst is pressing against a nerve or similarly sensitive structure. In this case report we seek to analyze one case of a painful soft tissue mass that was originally diagnosed as a ganglion cyst, had failed fine-needle aspiration, and was analyzed under MRI to be a case of "tenosynovitis." The goal is to understand that, all similarities aside, no two soft tissue masses are completely identical, and that it is important to obtain the correct diagnosis early in treatment, to render the appropriate care so that the symptoms do not become debilitating or impact the patient's activities of daily living.
\end{abstract}

\section{Introduction}

Ganglion cysts are benign soft tissue masses that can typically arise from tendon sheaths in the hands and feet, although are more common in the upper extremities [1]. These are benign in nature. When assessing ganglion cysts, their geographic location must be taken into careful consideration as many of these soft tissue masses, while otherwise harmless, may press against nearby neurovascular structures, causing secondary complaints of neuropathy and neuritis. Examples of peripheral nerve entrapments have been cited in the literature, particularly following back surgery [2]. Conservative treatments of ganglion cysts are previously described within the literature as well, which typically include options such as supportive, comfortable shoe gear with padding to help either cushion or off-load the affected area or fine needle aspiration [3]. It is known that ganglia tend to have a high rate of recurrence as well. Ganglion cysts are frequently seen along the dorsum of the foot, with many patients presenting with paresthesia distal to the mass, likely secondary to compression neuritis of sensory nerves adjacent to the ganglion [4]. Another differential to consider is a fibroma. Fibromas are benign tumors that can arise from the synovium of a tendon sheath, and usually occur around smaller joints such as the fingers or toes. These lesions are well circumscribed and lobulated, and histologically show presence of elongated thin-walled vessels or clefts, called slit-like spaces [5].

Advanced imaging can provide additional information regarding diagnosis of a soft tissue mass, and the other differential encountered in this case after an MRI read was tenosynovitis of the EHL tendon. When considering tendinopathy on MRI, one author mentions that tendinosis appearsas a "loss of the anterior concave or flat surface, fusiform thickening of the tendon, and increased signal intensity in the tendon" [6]. These findings were not mentioned within the official MRI read, though increased signal intensity around the tendon was appreciated.

\section{Case Report}

A 52-year-old male presented to clinic with a painful, palpable soft tissue mass noted to the anterior aspect of the left foot, immediately distal to the level of the ankle joint. He states that he noticed it occurred back in November 2016. He denies an inciting trauma, foreign body, or similar event. He states that the mass occurred suddenly and without warning.

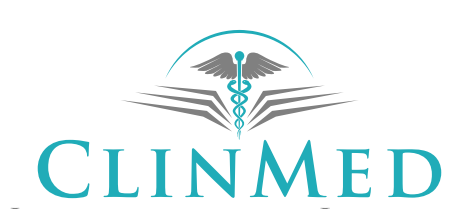

INTERNATIONAL LIBRARY

Citation: Atrushi K (2020) An Atypical Soft Tissue Mass on the Dorsum of the Foot: A Case Report. Int J Foot Ankle 4:040. doi.org/10.23937/2643-3885/1710040 
He relates that the mass has caused him pain that radiates down into his hallux and $2^{\text {nd }}$ digit of his left foot. He describes this pain as burning and tingling in sensation. He stated that overall, nothing seemed to improve the pain, and that he would have discomfort at random intervals during the day. Soft tissue masses have been documented to have lesions along the course of a single nerve, such as schwannomas, though there are few instances in literature where schwannomas were noted as a raised, palpable and visible soft tissue mass [7]. Given no prior history of gout, and limited clinical findings consistent with gout, other differentials may be considered such as neoplasm, indolent infection, or inflammatory synovitis [8]. While the patient was initially concerned for malignancy, it is noted that the majority of soft tissue masses are benign [9].

Upon clinical examination, a bounding dorsalis pedis pulse is visualized along the anterior surface of the foot, adding aneurysm of the dorsalis pedis into the differential. According to Berard, et al. aneurysms of peripheral arteries are rare, with an incidence rate of less than $1 \%$ and only 12 cases of true dorsalis pedis artery aneurysm have been reported in the literature [10]. Furthermore, both aneurysms and pseudoaneurysms have an association with traumatic injury or iatrogenic intervention, both of which are absent in this patient's clinical history [11]. A venous aneurysm is clinically observed as a non-pulsatile mass with shrinkage upon elevation and enlargement with dependency and this was not appreciated on clinical exam [12]. Duplex scan of the affected artery may provide additional information regarding patency and shape of vascular malformation, but this was not obtained [13].

\section{Discussion}

Ganglion cysts are relatively common and benign soft tissue masses encountered over tendon sheaths in the foot. This particular patient had some physical exam findings that could lead one to follow alternative diagnoses. The mass did not completely transilluminate under light, and the patient had a corresponding bounding pulse of the dorsalis pedis (so much so that the pulsing artery was visible under the skin), also making one consider the possibility of an aneurysm of the dorsalis pedis. Of note, the MRI showed increased signal intensity consistent with a possible tenosynovitis of the FHL tendon.

The patient was consented for excisional biopsy of the soft tissue mass to be preformed in the operating room. The patient tolerated surgery and anesthesia, and had no immediate post-operative complications, and no complications were noted on follow-up including up to 3 months after the surgery. The sample was sent to pathology for analysis, and came back as a vascularized fibroma with a blood clot inside (Figure 1, Figure 2, Figure 3 and Figure 4).

An initial possible diagnosis was an aneurysm of the dorsalis pedis artery, evident by the visualization of a

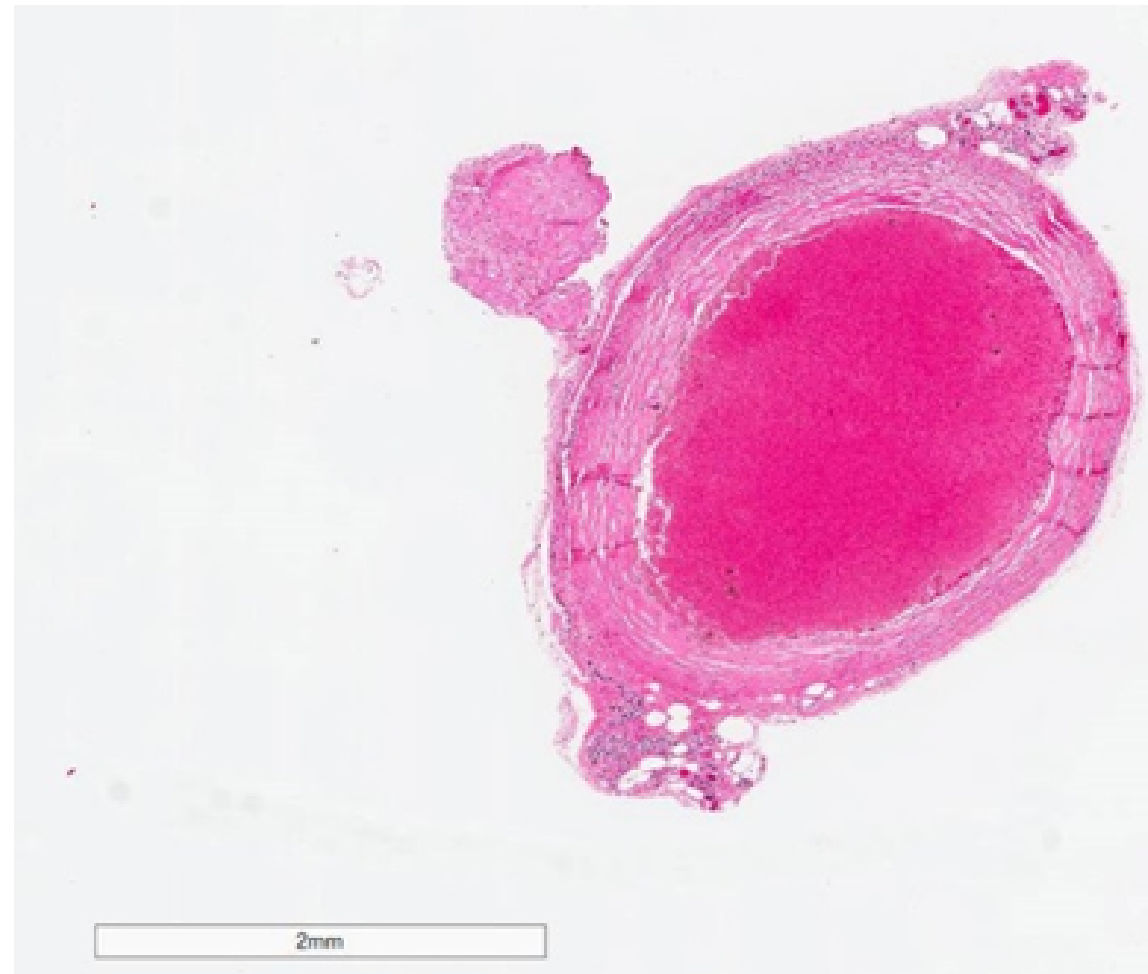

Figure 1: 1.4X magnification H\&E - Birdseye view of biopsy showing nodule of blood clot surrounded by fibrous wall. Definitive diagnosis of soft tissue masses should be made on histopathological examination [14]. The lesion appears well circumscribed. 


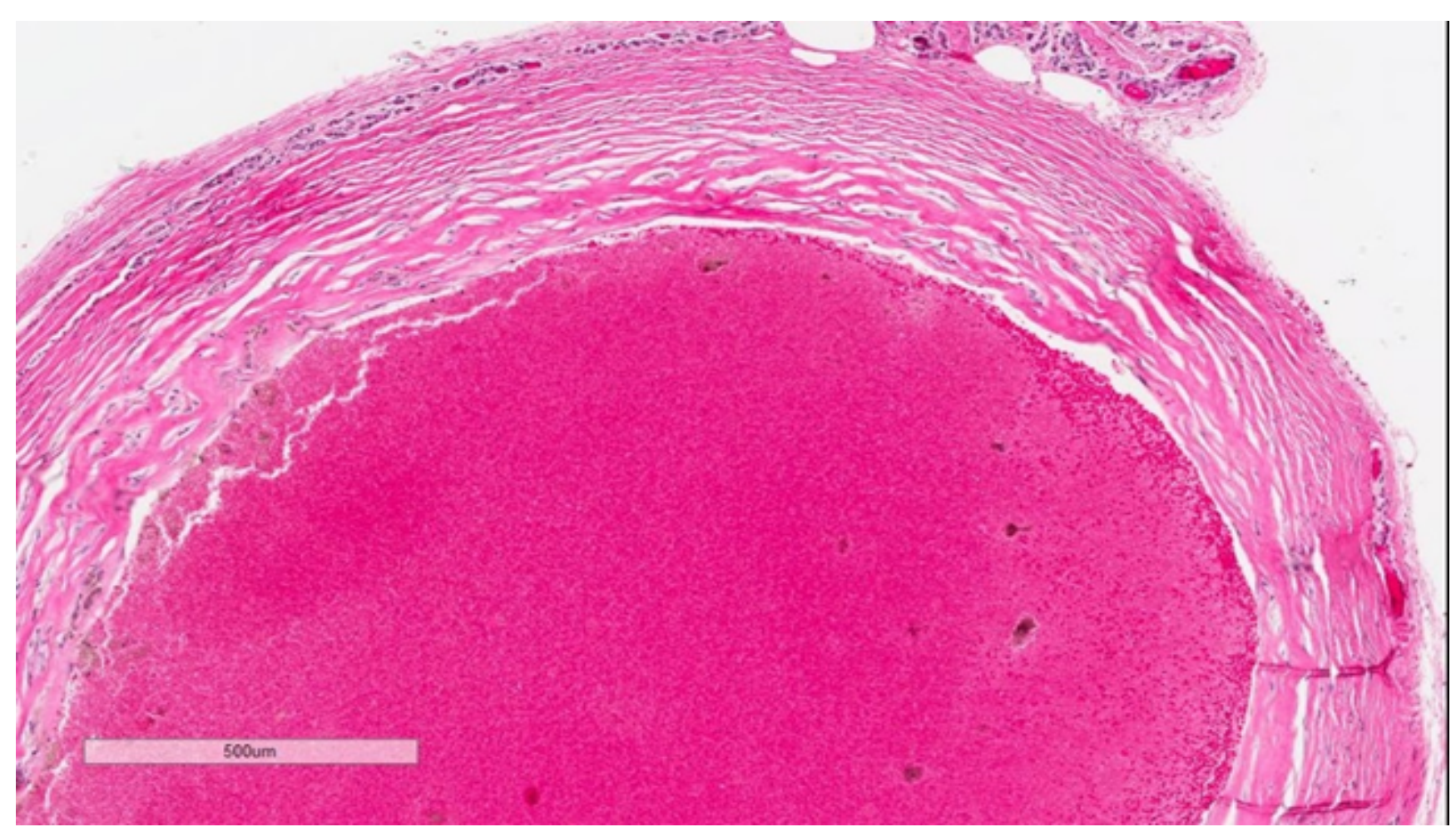

Figure 2: 5X magnification H\&E - Higher power of biopsy showing blood clot with focal hemosiderin laden macrophages surrounded by thick fibrous wall. The "slit-like spaces" can be appreciated along the outer wall, consistent with fibroma. Thick, fibrous septae can also be seen in giant cell tumors of tendon sheaths [15].

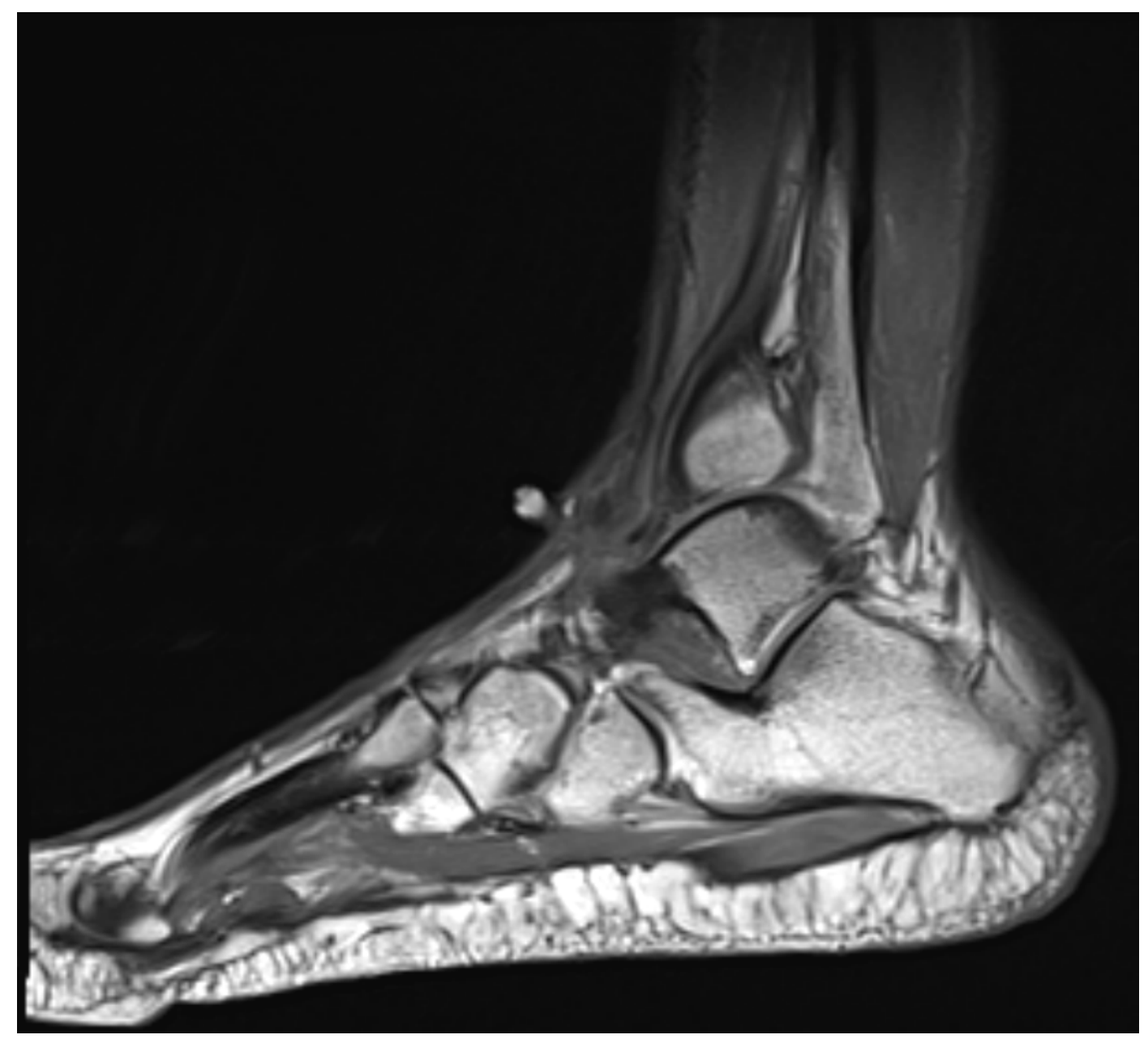

Figure 3: Sagittal T1-weighted MRI showing increased signal intensity at the subcutaneous tissue at the anterior aspect of the ankle. A fibroma localizing from the ankle joint capsule has been described by Ciatti, et al. [16]. 


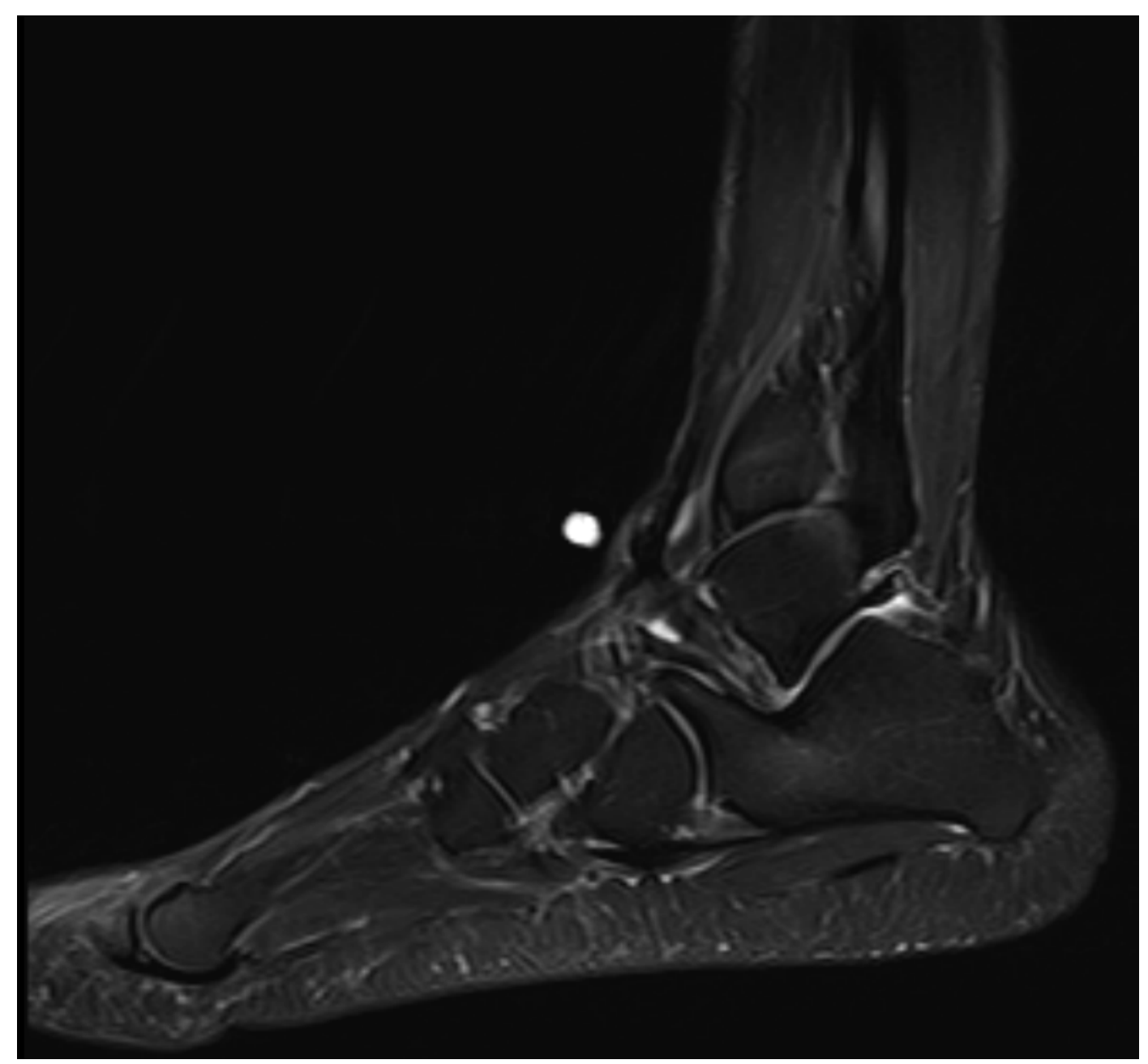

Figure 4: Sagittal T2-weighted MRI showing decreased signal intensity at the subcutaneous tissue at the anterior aspect of the ankle. Tenosynovitis would likely show increased uptake on this imaging modality [17].

pulsatile mass of soft tissue noted along the dorsum of the foot. It is possible see to an expansion of fibrous capsule as well, compressing adjacent soft tissue structures, such as the deep peroneal nerve. A lipomatous nature of the lesion is unlikely, as the excision did not show a white-yellow, homogenous structure consistent with lipoma or lipofibromatosis $[18,19]$. An intraneural ganglion cyst can be identified within the epineurium of peripheral nerves, including the tibial nerve, medial plantar nerve, superficial peroneal and sural nerves, though rarely within the deep peroneal nerve [20].

\section{Conclusion}

The authors present an unusual case of benign soft tissue mass that was diagnosed as a ganglion cyst on initial clinical evaluation, as tenosynovitis of the $\mathrm{FHL}$ on $\mathrm{MRI}$, and as vascular malformation of the dorsalis pedis artery on subsequent visit. The pathology report shows essentially a fibrotic walled-off blood clot. The authors hope to increase understanding of various differentials of soft tissue masses in the lower extremity, while also providing information about an unusual presentation of such an entity. It is important to keep in mind all likely differentials of any soft tissue mass, but to also understand and expect that, even with an accurate H\&P and advanced imaging, a soft tissue mass can not be diagnosed $100 \%$ without pathologic study.

\section{Financial Disclosure}

None reported.

\section{Conflict of Interest}

None reported.

\section{Acknowledgements}

The authors would like to thank Dr. Stephen Lau (Department of Pathology, Atlanta VA Medical Center) for his assistance.

\section{References}

1. Pontious J, Good J, Maxian SH (1999) Ganglions of the foot and ankle. A retrospective analysis of 63 procedures. J Am Podiatr Med Assoc 89: 163-168.

2. Ang CL, Foo LSS (2014) Multiple locations of nerve compression: An unusual cause of persistent lower limb paresthesia. J Foot Ankle Surg 53: 763-767.

3. Ahn JH, Choy WS, Kim HY (2010) Operative treatment for ganglion cysts of the foot and ankle. J Foot Ankle Surg 49: 442-445.

4. Lemont H, Sabo MA (2000) Origin of Foot Ganglion. J Am Podiatr Med Assoc 90: 256-257. 
5. Suzuki K, Yasuda T, Suzawa S, Watanabe K, Kanamori M, et al. (2017) Fibroma of tendon sheath around large joints: Clinical characteristics and literature review. BMC Musculoskelet Disord 18: 376.

6. Riley GM (2007) Magnetic resonance imaging in the evaluation of sports injuries of the foot and ankle: A pictorial essay. Sports Medicine of the Lower Extremity 97: 59-67.

7. Ly $P$ (2017) Solitary benign schwannoma of the foot presenting clinically as a ganglion cyst. A Case Study. J Am Podiatr Med Assoc 108: 340-343.

8. Vinson EN (2006) Clinical Images: A not-so crystal-clear ankle mass. Arthritis Rheum 54: 2706.

9. Reshadi H, Rouhani A, Mohajerzadeh S, Moosa M, Elmi A (2014) Prevalence of malignant soft tissue tumors in extremities: An epidemiological study in syria. Arch Bone Jt Surg 2: 106-110.

10. Berard X, Bodin R, Saucy F, Deglise S, Pailler A, et al. (2011) Current management of true aneurysm of the dorsalis pedis artery. Ann Vasc Surg 25: 1-4.

11. Vlachovsky R, Staffa R, Novotny T (2017) Pseudoaneurysm of the dorsalis pedis artery: Case Report and Literature Review. J Foot Ankle Surg 56: 398-400.

12. Freeman D, Randall DB (2001) Venous aneurysm of the dorsal venous arch. J Am Podiatr Med Assoc 91: 93-101.

13. McKee TI, Fischer JB (2000) Dorsalis pedis artery aneu- rysm: Case report and literature review. J Vasc Surg 31: 589-591.

14. Grover A, Nagaraj P, Joseph VM, Gadi D (2017) Unusual presentation of mycetoma of the foot: A rare case report. J Orthop Case Rep 7: 12-15.

15. Chen YU, Yu XC, Xu SF, Wang B (2016) Giant cell tumor of the tendon sheath originating from the ankle capsule: $A$ case report and literature review. Oncol Lett 11: 3461-3464.

16. Ciatti R, Mariani PP (2009) Fibroma of tendon sheath located within the ankle joint capsule. J Orthop Traumatol 10: 147-150.

17. Sakurai M, Kobayashi H, Kobayashi T (2007) Extensor digitorum longus tenosynovitis caused by talar head impingement in an ultramarathon runner: A case report. J Orthop Surg 15: 245-247.

18. Seagrave RA 3rd, Templeton KJ (2014) Soft tissue ankle mass in a 15-month-old girl. Clin Orthop Relat Res 472: 1058-1062.

19. Liu YL, Wang ML, Liu JY (2008) Presacral myelolipoma. Taiwan J Obstet Gynecol 47: 241-243.

20. Blitz NM, Amrami KK, Spinner RJ (2009) Magnetic resonance imaging of a deep peroneal intraneural ganglion cyst originating from the second metatarsophalangeal joint: $A$ pattern of propagation supporting the unified articular (synovial) theory for the formation of intraneural ganglia. J Foot Ankle Surg 48: 80-84. 